\title{
Wildfire Monitoring and Hotspots Detection with Aerial Robots: Measurement Campaign and First Results
}

\author{
Alberto Viseras*, Juan Marchal*, Marius Schaab*, Jordi Pages ${ }^{\dagger}$, and Laia Estivill ${ }^{\dagger}$
}

\begin{abstract}
Wildfires destroy thousands of hectares every summer all over the globe. Nowadays, wildfire prevention is typically done by fire fighters on foot scanning the areas in danger to detect potential hotspots, or by static surveillance systems. These solutions involve either a high human effort, which may also result in dangerous situations, or a high cost to deploy and maintain static surveillance systems. In contrast, aerial robots are a perfect fit for wildfire prevention, as they are able to scan an area autonomously to detect potential hotspots. A review of the available literature has revealed that analysis of hotspot imagery as seen from a drone perspective is absent. In this paper, we describe the first campaign that gathered thermal and visual images with a drone in multiple scenarios. Our findings indicate that even $15 \mathrm{~cm}$ hotspots could be easily identified from a drone.
\end{abstract}

\section{INTRODUCTION}

EU Project HEIMDALL, funded under Horizon-2020 Program, has been set to develop and test a Multi-Hazard Cooperative Management Tool for Data Exchange, Response Planning and Scenario Building [1]. One of the considered aspects within the project is the detection of wildfire hotspots with a swarm of aerial robots.

A review of the current sensing technology for wildfire detection and monitoring can be found in [4]. There also exist previous works in the literature that deal with wildfire monitoring [2], and with wildfire hotspots detection [3], [5]. On the one hand, both papers [2], [3] focus on the algorithm development for the cooperation among aerial robots. On the other hand, [5] developed wildfire detection algorithms using complex image processing techniques. Aforementioned works present outstanding results from the algorithmic perspective. However, developed algorithms were developed and tested using little actual hotspot data.

We strongly believe that the development of a hotspot detection algorithm requires an extensive amount of data to assure the correctness of developed algorithms. To this end, we carried out the first measurement campaign that targets systematic measurements of hotspots as viewed from a drone.

In the reminder of the paper, we first describe the measurement campaign setup in Sec. II. This is followed in Sec. III by first results obtained from data gathered during the campaign. We finalize this paper with conclusion and future work.

*All authors are with the Institute of Communications and Navigation of the German Aerospace Center (DLR), 82234, Oberpfaffenhofen, Germany \{alberto.viserasruiz, juan.marchalgomez, marius.schaab\}@dlr.de

${ }^{\dagger}$ Both authors are with Catalan Fire and Rescue Service Bombers Generalitat de Catalunya, Barcelona, Spain $\{j \cdot$ pagesc, laia.estivill\}@gencat.cat
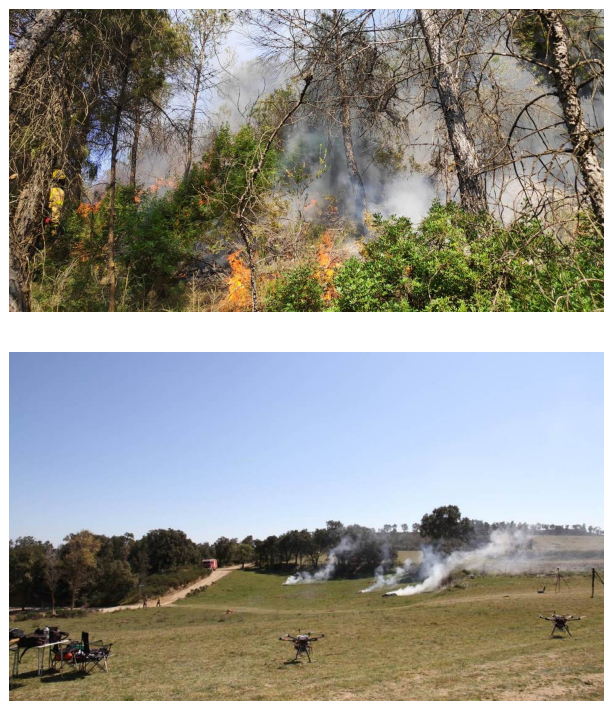

Fig. 1: Measurement campaign setup. Top: controlled burning of a forest during campaign's first day. Bottom: burning hotspots during an experiment run.

\section{Measurement Campaign Setup}

The measurement campaign took place from $18^{\text {th }}$ till $22^{\text {nd }}$ of March 2019 in Catalonia (Spain). The first day was dedicated to a controlled burn organized by the firefighting unit from Catalonia (see Fig. 1). The controlled burn consisted of low brush burning in the forest to prevent forest fires in the upcoming summer season. Using our drone platform consisting of three autonomously flying drones, we were able to collect actual geo-referenced fire data, in both visual and thermal infrared spectra.

Next two days of the campaign were dedicated to analysis of different wildfire hotspots. Specifically, multiple scenarios consisting of hotspots composed by different materials, such as wood, thatch, or sepiolite were prepared; different sizes of hotspots, ranging between $0.15 \mathrm{~m}$ and $1.5 \mathrm{~m}$ were tested (see Fig. 1). Also, different stages of hotspot dynamics starting from ignition to close-to-be-extinguished were inspected with cameras. In total, 37 different hotspots types were analyzed during 19 different flights. During measurements also different flight altitudes ranging between 30 and 130 meters were studied.

We carried out measurements with 3 different drones. The used drones were based on the DJI S900 platform that was modified appropriately to carry the sensing and data processing equipment (see Fig. 2). The drones are 


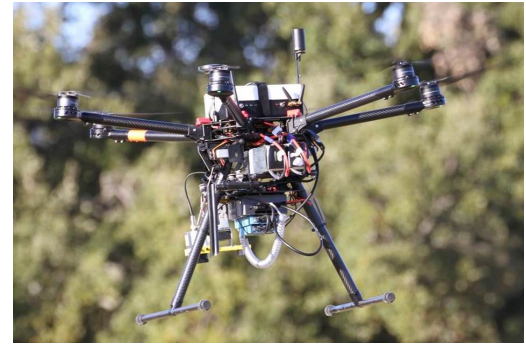

Fig. 2: Drone employed to gather data.

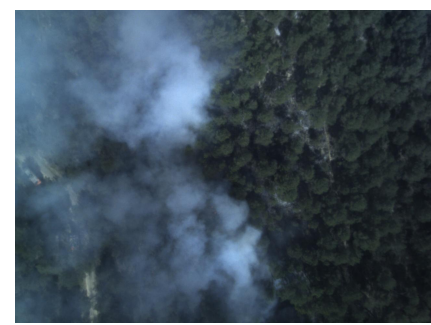

(a) Visual image

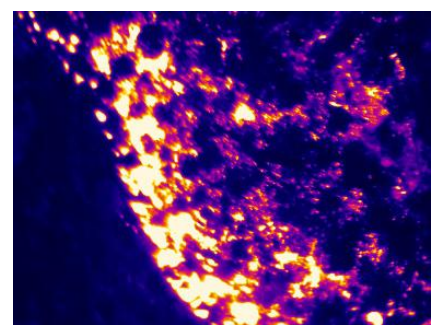

(b) Thermal image.
Fig. 3: Visual and thermal images taken from a drone during a controlled burn.

all equipped with a thermal and a visual camera, and a Global Positioning System Real Time Kinematic (GPS-RTK) system for precise positioning. Drones took geo-referenced images every 0.2 seconds. Pictures taken by the drone were saved on an on-board Intel NUC computer. The used visual camera is an mvBlueFOX-MLC202bC from Matrix Vision with a 82 degrees angle of view lens. The thermal camera is an Optris PI 400 with a $29 \times 22$ degrees angle of view lens. For the thermal cameras we collected data using three temperature range configurations: $[-20,100],[0,250]$ and $[150,900]$ degrees Celsius.

\section{Measurement Campaign Results}

In total, we collected $70 \mathrm{~GB}$ of image data from all experiments runs. In Fig. 3 we can observe a visual and a thermal image taken from a drone during the controlled burn. On the visual image we can observe the smoke caused by the fire, while on the thermal one we can clearly identify the fire front.

We also evaluated the ability of the camera to identify small hotspots. In Fig. 4 we depict two thermal images as taken by a drone flying at a height of 90 meters. We can see from the pictures that even a very small hotspot with a size of $15 \mathrm{~cm}$ can be easily identified.

In addition, we took advantage of the geo-referenced pictures to generate a mosaic. In Fig. 5 we show a mosaic of the gathered thermal images overlaid on a map.

\section{CONClusion And Future Work}

In this paper we presented the first measurement campaign to systematically gather data from wildfire hotspots. We took visual and thermal images with 3 different drones, equipped with a GPS-RTK antenna, flying at heights between 30 and

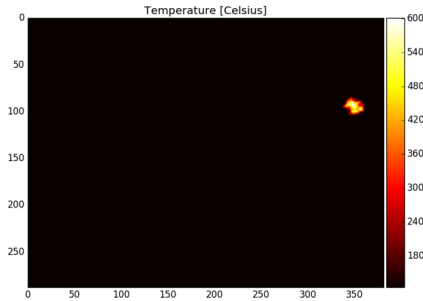

(a) 1 meter size hotspot.

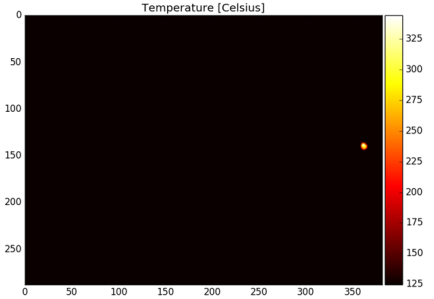

(b) 15 centimeters size hotspot.
Fig. 4: Thermal images of two distinct hotspots captured by a drone flying at 90 meters height.

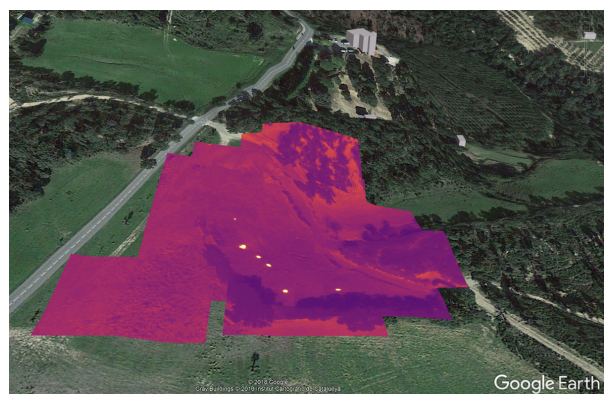

Fig. 5: Geo-referenced mosaic generated from thermal images taken by a drone while several hotspots were burning. Color scale represents floor's temperature, where light is coldest and yellow is hottest temperature.

130 meters. We also showed first results of gathered images from a controlled burn, a $1 \mathrm{~m}$ and a $15 \mathrm{~cm}$ hotspot. Our next step is to make the gathered data publicly available, which will highly impact research in wildfire monitoring and hotspot search with aerial robots.

Additionally, we aim to investigate the use of aerial robots in more complex scenarios. This includes the use of drones in the presence of other aerial platforms, and under a strong convective column caused by a fire.

\section{ACKNOWLEDGEMENT}

We would like to acknowledge the work of Edgar Nebot, Claudi Gallardo, Daniel Milla, Christoph Manss, and Thomas Wiedemann. This work was supported by European H2020 project HEIMDALL.

\section{REFERENCES}

[1] "HEIMDALL - Multi-Hazard Cooperative Management Tool for Data Exchange, Response Planning and Scenario Building," http://heimdallh2020.eu/.

[2] K. D. Julian and M. J. Kochenderfer, "Autonomous distributed wildfire surveillance using deep reinforcement learning," in 2018 AIAA Guidance, Navigation, and Control Conference, 2018, p. 1589.

[3] L. Merino, F. Caballero, J. R. Martínez-De-Dios, I. Maza, and A. Ollero, "An unmanned aircraft system for automatic forest fire monitoring and measurement," Journal of Intelligent \& Robotic Systems, vol. 65, no. 1-4, pp. 533-548, 2012.

[4] R. Allison, J. Johnston, G. Craig, and S. Jennings, "Airborne optical and thermal remote sensing for wildfire detection and monitoring," Sensors, vol. 16, no. 8, p. 1310, 2016.

[5] C. Yuan, Z. Liu, and Y. Zhang, "Uav-based forest fire detection and tracking using image processing techniques," in 2015 International Conference on Unmanned Aircraft Systems (ICUAS). IEEE, 2015, pp. 639-643. 